\title{
Social interconnection as a factor of the effectiveness of Second Chance Schools. A case study from a Detention Center in Greece
}

\author{
Amalia Stoumpou ${ }^{a}$, Natassa Raikou ${ }^{b}$ \\ a Hellenic Open University, amalia.stoumpou@gmail.com \\ ${ }^{b}$ University of Patras \& Hellenic Open University, araikou@upatras.gr
}

\begin{abstract}
This paper refers to the importance of social interconnection between a Second Chance School (S.C.S.) in a detention center and the local community. The research was based on the qualitative approach and on the tool of the semi-structured interview for the collection of research data. Six trainers, eight trainees and the Director of the 2 nd S.C.S. of Patras, were the sample of the research, while the technique of content analysis was chosen for the analysis of the data. The results of the survey show that there is a positive effect of the school's openness in achieving its educational objectives. In particular, social interconnection actions contribute to personal development, professional and educational vigilance, the acquisition of social skills, the change of attitudes and, to a lesser extent, to post-internment re-socialization. In addition, in order to improve the effectiveness of these actions, it is proposed that they are organized on a more stable and regular basis, as well as to focus mainly on vocational guidance.
\end{abstract}

Keywords: prison education, social interconnection actions, school-society relationship, Second Chance Schools in prisons

\section{Introduction}

The central role of social interconnection of S.C.S.s is increasingly being discussed nowadays due to its great importance. The statutory goal of S.C.S.s is the development of students' personal and social competences, as well as improving their employment opportunities (Article 1.2, Presidential Decree No. 2373/2003). This issue acquires special importance in S.C.S.s in detention centers taking into account that prison education is considered as one of the means that promote re-socialization during confinement, while increasing the possibility for social and professional reintegration after release.

In order to examine the significance of social interconnection between S.C.S.s in detention centers and the local community, this article provides a conceptual clarification and, additionally, an overview of theoretical approaches related to this subject, while using a research example in order to draw conclusions on this subject.

In particular, this paper initially situates the concept of social interconnection of schools within a wider concept. Especially, it stands on social interconnection of S.C.S.S, as well as the connection with local community through educational programs in detention centers and also considers the conditions for effective education for prisoners. Following these introductory sections, a case study of the $2^{\text {nd }}$ S.C.S. of Patras is presented. The purpose and the methodology of the study are described, followed by an analysis of the findings from interviews. Drawing on these results, it concludes with a summary of findings and a discussion 
of possible implications in conjunction with theoretical approaches and the relevant literature review.

\subsection{Key terms and theoretical framework}

Since the inmates are in an environment that by its nature does not offer opportunities and further options, school staff is actively engaged in developing networks of partnerships with local community. To better understand social interconnection of S.C.S.s in detention centers and what fosters their development, it is crucial to study the forms of community partnerships chosen (such as visits by community members, agencies, organizations), but also their impact on the effectiveness of the school unit.

In the present study, social interconnection is defined as any meaningful cooperation with community bodies, that focuses on students, and supports the cognitive, social, emotional, and intellectual well-being of them, directly or indirectly (Willems \& Gonzalez-DeHass, 2012). This connection incorporates partnerships with educational and research bodies, businesses, NGOs, health care organizations, cultural and entertainment bodies, volunteer groups or individuals that promote development and social well-being. Specific examples of such actions include (but are not limited to) recreational activities related to the school, visits in the context of projects, scientific workshops, experiential actions, speeches and presentations during school festivities and voluntary actions undertaken by the school.

The theoretical focus on the issue of social interconnection between S. C. S.s in detention centers and the local community, is still in the early stages of development. Most of the research that has been done in our country and abroad, mainly investigates the social interconnection of schools in general without focusing on S.C.S.s and especially those schools that are located inside a detention center. Consequently, even the issue of effectiveness of these actions has not so far produced significant research, with researchers generally highlighting the key components for the more effective organization of educational programs in detention centers.

\subsubsection{Principles of effective prison education}

In order to be effective, prison educational programs require special attention to be given to their planning, implementation and evaluation of their operation. Existing literature on the effectiveness of prison educational programs does not provide information on program characteristics - such as curricula, dosage, and staffing - that is necessary to determine best practices (MacKenzie, 2008).

Dimitrouli \& Rigoutsou (2017), claim that on the basis of the characteristics of the trainees in prison, the conditions prevailing and what education for prisoners symbolizes, prison education can be effectively organized, if the educational program is well structured and arranged, educational goals are clear and linked to the needs of prisoners, and highlights the special qualifications required by trainers.

Similarly, MacKenzie (2008), referring to correctional training in general and not specific to education, emphasizes additional efficacy parameters such as the qualifications and the training of the staff involved, the importance of program dosage and the appropriate staff-tostudent ratios. She concludes that the most effective programs emphasize individual rehabilitation through skills building, cognitive development, and behavioral change. These program models should be based on a clear theoretical framework, as well as the program elements and methods should be grounded in research. 
Other researchers, such as Cullen and Gendreau (2000), underline factors that focus mainly on the benefits learners receive after release. In their outline of effective educational program design, they note, in addition to the above, the importance of acquiring skills that enhance employability, as well as monitoring and evaluating the educational process and its results. They consider that without careful planning, implementation and evaluation, it is impossible to know whether and how the programs are effective in order to maintain or improve its effectiveness.

\subsubsection{Social interconnection of S.C.S.S}

In our country, the primary direction of the openness of S.C.S.s is highlighted in the statutory framework for their organization and operation: "S.C.S.s collaborate and seek to collaborate with all relevant social bodies, both to raise awareness of the social groups to which the school program is addressed, and to implement the curriculum" (Article 3, Presidential Decree No. 2373/2003, issue of Greek Government Gazette [FEK] B' 1003/22-7-2003). The same provision is also found in Article 3 of Presidential Decree No. 260/2008 on the organization and operation of S.C.S.s (issue of Greek Government Gazette [FEK] B' 34/16-1-2008). In addition, it is pointed out in Article 9 that the creation of cooperation networks between local authorities and social bodies to support the operation of schools is necessary in order to achieve the objective of S.C.S.S.

Vergidou (2016), in her survey, emphasizes that in the majority of S.C.S.s significant educational actions were carried out. Especially in schools operating in detention centers, the Directors developed collaborations with institutions such as high schools, universities, colleges and partnerships with various institutions such as the Eugenides Foundation etc. Antoniadou (2014) outlines that trainers and trainees are involved in programs that favor the opening of their school to society, bringing the trainers in contact with activities that allow the school to collaborate with organizations outside its boundaries.

In line with the above the results of the research of Anagnou and Vergidis (2008) suggest that at the initiative of the Directors, the S.C.S.s develop multi-faceted cooperation with local community bodies. However, according to the same researchers, the development of a network of cooperation with the local community, dictated by the institutional framework mentioned above, manifests itself unilaterally, having as a starting point the S.C.S.S. Interconnection initiatives come mainly from the Directors of the S.C.S.S and are being implemented either to a greater or lesser extent, bearing witness to the lack of a wider plan on the part of the local community, which would combine appropriate educational, cultural, productive and political activities.

\subsubsection{Social interconnection with local community through educational programs in} detection centers

The active role of local society is considered to be decisive in education. It is generally accepted that this interconnection is particularly useful and necessary, since it aims to fulfill multiple needs of prisoners such as employment, education, physical and mental health, family reconnection and social reintegration in general.

Improving and, therefore, expanding educational programs is a policy issue that requires strategic planning and is built through the formation of partnerships with a variety of bodies (Lawrence et al., 2002). Various partners, with different orientation, related to employment, education, health, culture and social welfare, businesses, NGOs, etc., can agree on a common goal, that of addressing the needs of prisoners, offering them motivation through designed 
educational actions. Literature can identify types of programs that provide full coverage of the needs of prisoners (such as S.C.S.s), as well as programs designed solely to serve some specific needs of prisoners such as education, employment, counseling, etc. (Presidential Decree No. 2357, issue of Greek Government Gazette [FEK] B' 327/8.2.2019).

In our country, the first, as well as the sole, official agency providing ex-prisoners with "aftercare" services to be established in Greece is "EPANODOS», a non-profit legal entity of private law supervised by the Greek Ministry for Citizen Protection. According to its founding act (Presidential Decree No. 300/2003), it aims at prisoners and ex-prisoners vocational support, as well as at the preparation and promotion of their social reintegration in a broad sense.

Furthermore, given that the content of education includes sporting and cultural activities, the Strategic Plan for the Penitentiary System in our country, points out as a key action of the 4th strategic goal, cultural activities, in cooperation with local cultural institutions. For example, collaborations have been developed with the National Theater for the creation of a theater group, with the National Opera for the implementation of educational programs of music and dance, as well as with the Ministry of Culture for the construction of a permanent museum exhibition. Apart from the cultural axis, sports programs in cooperation with the General Secretariat of Sports are already being implemented and are expected to expand to more detection centers.

In addition to the practices mentioned above, researchers such as Tsilikis and Gakou (2018) propose the design of a coherent reintegration framework, which utilizes existing programs and tools, and combines collaborative entrepreneurship, with social and employment reactivation of prisoners. In this direction, the interconnection of prison and local society is established, as the prisoners prepare themselves to take on roles other than those required by the incarcerated environment.

In conclusion, it is obvious that social reintegration is a question of "collective effectiveness" of the community that welcomes people released from prison (Visher \& Travis, 2003). They need - in addition to their personal effort - facilitation and supporting by local community towards their effort. Preparation for this purpose is clearly starting from the incarcerated life and the S.C.S., which can play a primary role in the implementation of that objective.

\section{Method}

The purpose of this survey is to explore the views of trainers, trainees and the Director of the 2nd S.C.S. of Patras within the detention center of Agios Stefanos, on the effectiveness of social interconnection with local community. More specifically, it is a case study, that analyzes how the interconnection between school and society satisfies the pedagogical, educational and social role of the school and the corresponding needs of the trainers.

In terms of the methodology used, the qualitative approach was chosen, which allows a detailed and in-depth approximation of the subject. Quality researchers focus on processes and results, as they seek to build a holistic and comprehensive picture of a subject, conducting the study in its physical context (Creswell, 2011). Additionally, as Creswell argues (2011, p.17), qualitative research is best suited to addressing an issue for which the available literature provides little information and needs participants' views to be demonstrated through investigation.

Through semi-structured interviews with trainees, trainers and the school Director, attempts are made to highlight the views of all participants of the school unit regarding the issue. The 
semi-structured interview is a form of interview that moves between a strictly structured interview and a free discussion. It allows to modify the content and the order of the questions depending on the respondent, as well as further penetration on some issues with participants that are considered well-qualified (Isari \& Pourkos, 2015).

The sample of this survey consists of six (6) trainers (of which 3 are school trainers in the year 2019-2020, while the remaining three worked in the school year 2018-2019), eight (8) trainees (7 in the school year 2019-2022 and 1 in the school year 2018-2019) and the Director of the school in the school year 2019-2020. This is a set, which constitutes a sample of convenience for the present investigation, which almost certainly does not represent the trainees, trainers and directors of the S.C.S.s in detection centers in their entirety. However, this research is a first approach to the issue in our country, which, in no way, aims to generalize the results. Therefore, the data resulting from the selection of a convenience sample can provide the basis for further research or allow for correlations with already existing findings in the field (Bryman, 2017).

In order to derive meaning from the data collected from the semi-structured interviews and answer the research questions, content analysis was used. It is a method of systematic evidence study that focuses mainly on the expressed ideas and the obvious content of texts and less on their expression. Belerson (as cited in Bryman, 2017) argues that, content analysis is a research technique for objective, systematic and quantitative description of the manifest content of communication.

\section{Findings}

The results of the survey highlighted information and the participants' views on the social interconnection actions implemented in the specific S.C.S. In particular, with regard to the social interconnection actions offered in the 2 nd S.C.S., the following categories emerged after the analysis of the data according to the thematic area of interest:

1. Education and research bodies

2. Cultural and entertainment bodies

3. Health, safety and social reintegration bodies

4. Employment agencies and professionals

5. Volunteering and environment

6. Individuals

The findings of the survey show that the initiative of their organization is collectively taken by the Director and the school's trainers in cooperation with the Social Service of the detention centre, in order to issue the necessary permits, as characteristically stated by the School Director in the following passage, while it is also noted that the degree of initiatives that each school can take vary, depending on its management and the teachers' association:

"Social interconnection actions are organized other times collectively by the Director, who is responsible for the pedagogical course of the school unit, and the Teachers' Association, and other times by the trainers, with prior information from the Director and the Association, and are included in the subject they teach." (Director)

As regards views on the forms of social interconnection already implemented and considered effective, cultural actions are distinguished. A common perception, mentioned by four of the six trainers, the Director and three of the eight trainees, is that they distinguish actions that 
their thematic area focuses on culture. The content of this type of actions included dance, music, constructions (digits, ceramics, etc.), cinema. During their implementation, as mentioned, they had the opportunity to work creatively - forgetting for a while the plight of internment, to be entertained, to participate actively and to develop new skills or to cultivate what they already know. It should also be noted that two of the trainers' report actions in the context of cultural activities but emphasize that through them the trainees had the opportunity to give their impression to the outside society, which enabled their resocialization and extroversion.

The Director considers that of the actions already offered to the school, particularly effective are those that offer some certification of the knowledge they provide, which can be used as a qualification both during their confinement and after their release. He characteristically states:

"The actions that have the greatest impact and acceptance on our students are those from which they will be granted some certification, which may be useful to them when serving their sentence or after their release."

This view is not expressed by the trainers but is in line with what four of the eight trainees have said, who distinguish actions that focus on health and safety issues. The trainees consider that by participating in actions of this type, such as those organized with the participation of the Red Cross (which offered certification) and the Fire Brigade, they contributed to developing skills, gaining knowledge, expanding their skills and being able to offer assistance, which will have an impact both within the detention centre and in the "outside" society.

Interestingly, none of the trainees mentioned actions in which they participated and focus on vocational guidance. Two of the six trainers and the school Principal stand out in such actions. As they point out, they are suitable for the creative use of their time, but mainly for the cultivation and development of skills that will be useful after their release, in order to maintain their contact with the needs of the labour market.

Regarding the benefits of social interconnection actions, the findings of the research show that through those actions the teaching of cognitive subjects is complemented, new knowledge is acquired in various subjects and especially in those of particular concern to learners such as culture, health, professions. In addition, experienced scientists contribute to updating the knowledge already acquired before internment, especially in scientific and professional matters.

With regard to the skills acquired through practical openness and participation in it, the respondents gave weight to the development and cultivation of skills already acquired, to the discovery of new aptitudes and areas of interest, but above all to the acquisition of social skills. As regards the latter, both trainees and trainers stress the development of cooperation, organizing, targeting, decision-making and social intelligence capacities within the framework of action plans and activities involving local community bodies.

Nevertheless, the most important benefits of the social interconnection of S.C.S.s are found in the part of the attitudes cultivated. It should be noted that the actions support the resocialization of prisoners attending school, because within them, they come into contact with people who socialize with them equally. It is estimated that learners derive psychological benefits from participating in enjoyable activities, thus becoming more communicative and extroverted. They are easier to open up to those around them, creating relationships of trust. They also acquire a collective consciousness and function as a group. Trainees also detect 
changes at the individual level, drawing on self-confidence and self-esteem through actions. They reflect, remove stereotypes and accept school more as a social institution.

As regards the benefits to the relationships developed between the trainees and trainers engaged in actions involving social interconnection, these are visible on both sides. Both consider that the relations between them are tightened and become more equal, thus developing confidence and creating a pleasant and creative climate. The impact of the actions on the relationships between the trainees is also visible. This lies in principle with the better acquaintance of each other but above all in the possibility of cooperation and the creation of groups that promote, on the one hand, the exchange of knowledge and experience and, on the other hand, the motivation and encouragement of each other in the creative process.

Focusing on the potential benefits of post-release actions, the research findings show that it is difficult to know precisely how and to what extent the benefits of education in a Detention Centre affect the long-term, often, life outcomes of prisoners after confinement. This difficulty is even more evident in this study, which attempts to approach more specialized research fields. As research progresses in educational structures within detention centers, more safe conclusions are likely to be drawn linking the openness of education to a better life after incarceration.

On proposals to enhance the effectiveness of actions, all participants refer to issues relating to improving the organizing of actions. Joint proposals that can contribute to improving the effectiveness of actions are relevant to the duration, number of meetings and role of logistics. Trainers and trainees are also proposing a stable network of partnerships with the local community, with trainers pointing out in particular that this could operate within an established framework, so that social interconnection initiatives come from the institutions themselves and are not entirely dependent on the Director and the school's teaching staff.

Another common assessment to improve the effectiveness of the actions is to target them on issues that will concern the trainees after their release. It is reported that an action can be more effective when focusing on professional rehabilitation. In addition to the targeting of actions in the professional reintegration of prisoners, the issue of social reintegration is also mentioned. Trainers and trainees suggest that this could be prepared through actions, during their internment, if the actions offered can allow learners to make their mark on outside society. Trainers say this could arise in the context of actions, from the participation of trainees in cooperative schemes of productive purpose, such as social enterprises, which could last after their confinement. Characteristic is the following excerpt from an interview with a school trainer:

"The outflows I think are very important and could be done in the context of a social enterprise perhaps not only with an ultimate professional purpose but a social purpose. Again, when students feel they are producing something and offering and producing it for the outside, which means that when they come out, they will have something ready, there will be some imprint of them outside. From what we have read there are several such moves in some other countries."

\section{Conclusions}

This research highlights the effectiveness of social interconnection in the 2 nd S.C.S. of Patras, which operates within the detention centre of Agios Stefanos. It is noted, as in the surveys of Anagnou \& Vergidis (2008) and Vergidou (2016), that in schools operating in a detention centre, the Directors develop manifold collaborations and carry out remarkable actions with 
local community bodies. The findings of the survey show the positive impact of the school's openness on the achievement of its educational objectives and seem to confirm what has been mentioned in the existing surveys on the benefits of education in a Detention Centre in general, but also especially on the benefits of the openness of educational structures.

Social interconnection actions contribute more to the personal development, as well as the professional and educational vigilance of learners within them (Dimitrouli \& Rigoutsou, 2017). Moreover, the very nature of openness that bridges the "outside" with the "inside" lends a transformative character to educational function on a psychological and especially social level. As far as the psychological part is concerned, the sense of carefreeness from participating in pleasant activities is stressed by all the respondents. Accordingly, referring more generally to the training provided in S.C.S.s, many researchers (Dimitrouli \& Rigoutsou, 2017; Vergidis, etc., 2007; Tewksbury \& Stengel, 2006), note that education motivates the prisoner to creatively utilize his free time, significantly reducing his psychological burden from confinement.

On a social level, almost all interviewees referred to the strengthening of socialization and extroversion within prisons as well as re-socialization through contact with the outside world (Dimitrouli \& Rigoutsou, 2017; Brazzell et al., 2009). A common finding of the majority of trainers and trainees is that they open up, build relationships of trust and feel that they can be useful to those around them (Michelle et al., 2001; Brazzell et al., 2009).

However, with regard to the potential benefits of post-release actions, the general conclusions from the responses given in the interviews are in agreement with those of the existing studies on the results of education in general in a Detention Centre after release, in that these benefits exist but are not easily visible and detectable (Bushway \& Reuter, 1997; Lawrence et al., 2002).

In order to improve the effectiveness of actions, it is proposed that they should be organized on a more stable and regular basis, as well as their focus should mainly be on vocational guidance, issues raised by other researchers who generally refer to the educational programs offered in prisons (MacKenzie, 2008; Dimitrioulis \& Rigoutsou, 2017). In addition, it is stated that trainees can prepare their professional rehabilitation during their confinement if they are involved in actions through which they will be able to go out on their own through an outflow system (Tsilikis \& Gakou, 2018).

Finally, trainers and trainees consider that the actions would have been more effective if their implementation had been formally planned, organized and established, following research and diagnosis of the needs of the trainees. In a broader context of education in a Detention Centre, Lawrence et al. (2002) consider that the improvement and expansion of education programs is built by establishing relationships with a variety of actors and is a policy issue requiring strategic planning. More specifically, surveys concerning prison S.C.S.s show the lack of a broader plan on the part of the local community (Anagnou \& Vergidis, 2008), while it is necessary for the state to take more initiatives in relation to the interconnection of the school (Kofini, 2017).

As regards the limitations of research, the research approach, the research strategy and the sampling method chosen constitute a restrictive element with regard to the generalization of its findings, since an attempt was made to understand various aspects of the effectiveness of the actions in a context that concerns only the specific school. However, as has already been mentioned, the field under consideration has been little explored in our country, so the research carried out is essentially a first approach to the subject. 
Case studies like this in other schools, as well as a larger range of surveys with a richer and random sample, in more than one prison school, could confirm or disprove the findings of this research. In any case, however, they would enable its conclusions to be broadened and enriched. So future researchers seeking to address the issue of social interconnection and its effectiveness in the education of prisoners, have a wide range of research fields that gather all the evidence that has emerged from the results of the work.

In particular, there is a need to understand more deeply the concept of social interconnection both in terms of its content and in terms of its implementation. One of the research requests arising as a result of this study could be the identification of those elements of outside society that need to be connected to the prison society. What would be their direction and for what purpose in particular? The consequence of this issue is to further study the effectiveness of social interconnection actions in order to investigate which of them are effective and how. An integrated approach could identify whether the limits of this effectiveness stop within the school or extend beyond prison or even after release. Another level of study concerns the expressions of effectiveness in human relations, personal development, vocational guidance and social reintegration of learners. From then on, a wide range of variables involved in this research can be explored so that future researchers can formulate more clear correlations.

\section{References}

Anagnou, E., \& Vergidis, D. (2008). Second Chance Schools' action strategies. The institutional framework and the contribution of Directors. Adult Education, 13, 11-18. [in Greek]

Antoniadou, Th. (2014). The education of prisoners in Greece: the example of the operation of prison S.C.S. and the leadership style of these schools. Master thesis, University of Thessaly. [in Greek]

Vergidis, D., Asimaki, A., \& Jintzidis, A. (2007). The training of prisoners. The Second Chance School at Korydallos Prison. Arethas, IV,61-89. [in Greek]

Vergidou, A. (2016). The Directors of Second-Chance Schools. Their contribution to the effectiveness and culture of the school unit. Master thesis, University of Patras. [in Greek]

Brazzell, D., Crayton, A., Mukamal, D. A., Solomon, A. L., \& Lindahl, N. (2009). From the Classroom to the Community: Exploring the Role of Education during Incarceration and Reentry. The Urban Institute, Justice Policy Center. Retrieved January 20, 2020 from: https://files.eric.ed.gov/fulltext/ED508246.pdf

Bryman, A. (2017). Methods of social research. (transl. P. Sakellariou, ed. A. Aidine). Athens: Gutenberg. [in Greek]

Bushway, S. D., \& Reuter, P. (2003). Labor markets and crime risk factors. In L. W. Sherman, D. P. Farrington, B. C. Welsh, \& D. L. MacKenzie (Eds.). Evidence-based crime prevention (pp. 212-254). New York: Routledge.

Creswell, J.W. (2011). Research in education. Design, conduct and evaluation of quantitative and qualitative research (transl. N. Kouvarakou, ed. H. Jorbatzoudis). [in Greek]

Cullen, F. T., \& Gendreau, P. (2000). Assessing correctional rehabilitation: Policy, practice, and prospects. Criminal Justice, 3(1), 299-370.

Dimitrouli, K., \& Rigoutsou, E. (2017). Guide for Second Chance Schools Educators in Detention Centres. Athens: Ministry of National Education Research and Religious Affairs, General Secretariat for Lifelong Learning and Youth. [in Greek] 
Michelle, F., Torre, M. E., Boudin, K., Bowen, I., Clark, J., Hylton, D., Martinez, M. M., Roberts, R. A., Smart, P., \& Upegui, D. (2001). Changing: The Impact of College in a MaximumSecurity Prison. New York: The Graduate Research Center of the City University of New York.

Isari, F., \& Rourke, M. (2015). Qualitative Research Methodology. Athens: Association of Greek Academic Libraries. [in Greek]

Kofini, E. (2017). Addressing the social exclusion of prisoners through their participation in Second Chance Schools. Master thesis, Hellenic Open University. [in Greek]

Lawrence, S., Mears, D. P., Dubin, G., \& Travis, J. (2002). The practice and promise of prison programming. Washington: Urban Institute, Justice Policy Center.

MacKenzie, D. L. (2008). Structure and components of successful educational programs. Reentry Roundtable on Education, John Jay College of Criminal Justice, New

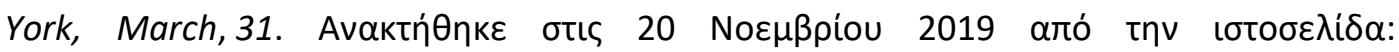
http://johnjay.jjay.cuny.edu/files/DorisMackenzie Final.pdf

Tewksbury, R., \& Stengel, K. M. (2006). Assessing correctional education programs: The students' perspective. Journal of Correctional Education, 13-25.

Tsilikis, H., \& Gakou, E. M. (2018). Social entrepreneurship and correctional. Proposed model for the reintegration of prisoners and released persons in Greece. Social Policy, 9, 88-110. [in Greek]

Visher, C. A., \& Travis, J. (2003). Transitions from prison to community: Understanding individual pathways. Annual review of sociology, 29(1), 89-113.

Willems, P., \& Gonzales-DeHass, A. (2012). School-community partnerships: Using authentic context to academically motivate students. School Community Journal, 22(2), 9-30.

\section{Corresponding author:}

Amalia Stoumpou, Hellenic Open University, amalia.stoumpou@gmail.com 